\title{
Relação entre diabetes e sintomas depressivos
}

\section{Relationship between diabetes and depressive symptoms}

\author{
João Fernando Marcolan' • Juliana Vallim Jorgetto² Giovanna Vallim Jorgetto $^{3}$
}

\begin{abstract}
RESUMO
Objetivo: Relacionar prevalência de depressão associada ao quadro de Diabetes Mellitus tipo 2, de população adulta de cidade do sul de Minas Gerais. Método: Estudo de análise documental, descritivo, retrospectivo de campo, em prontuários físicos/ eletrônicos do e-sus de indivíduos com Diabetes Mellitus tipo 2. Utilizado instrumento com informações sociodemográficas e clínicas relativas ao diabetes e sintomas depressivos. Análise de dados por média, desvio padrão, teste qui-quadrado $(p<0,05)$ e correlação de Pearson $(r>0,5)$. Resultados: Levantados 317 prontuários de indivíduos com diabetes tipo 2 ( $10,40 \%$ da população) e destes, analisados 226 prontuários $(30,08 \%)$ que apresentaram sintomatologia depressiva correlacionada. Média de idade 63 anos ( $d p=5,44)$, maioria sexo feminino (64,66\%), faixa etária 50/70 anos. Sintomas depressivos foram mais prevalentes nas mulheres (31,7\%), com renda mensal até dois salários mínimos, $42,08 \%$ eram aposentados e 7I,68\% apresentaram tempo de doença entre 5 a mais de 20 anos. Conclusão: Prevalência de diabetes tipo 2 acima da média nacional em população geral, com elevada prevalência de co-morbidade como o quadro depressivo. Deve-se realizar planejamento e intervenções específicas para a problemática por parte dos gestores municipais. Estudo contribui para revelar situação de prejuízo à saúde que não tinha sido detectado.
\end{abstract}

Descritores: Diabetes Mellitus; Diabetes Tipo 2; Depressão; Sintomas Depressivos; Epidemiologia; Morbidade.

\begin{abstract}
Objective: To relate the prevalence of depression associated with Diabetes Mellitus type 2, in an adult population in a city in southern Minas Gerais. Method: Study of documentary, descriptive, retrospective field analysis in physical / electronic medical records of the e-sus of individuals with type 2 diabetes mellitus. An instrument with sociodemographic and clinical information related to diabetes and depressive symptoms was used. Data analysis by mean, standard deviation, chi-square test $(p<0.05)$ and Pearson correlation $(r>0.5)$. Results: We surveyed 317 records of individuals with type 2 diabetes ( $10.40 \%$ of the population) and from these, 226 records $(30.08 \%)$ with correlated depressive symptoms were analyzed. Average age 63 years $(S D=5.44)$, mostly female $(64.66 \%)$, age $50 / 70$ years. Depressive symptoms were more prevalent in women (31.7\%), with monthly income up to two minimum wages, $42.08 \%$ were retired and $71.68 \%$ had a disease duration between 5 and over 20 years. Conclusion: Prevalence of type 2 diabetes above the national average in the general population, with a high prevalence of comorbidity as the depressive condition. Planning and specific interventions for the problem should be carried out by municipal managers. Study contributes to reveal situation of health damage that had not been detected.

Keywords: Diabetes Mellitus; Type 2 Diabetes; Depression; Depressive Symptoms; Epidemiology; Morbidity.
\end{abstract}




\section{INTRODUÇÃO}

O Diabetes Mellitus (DM) configura um problema de saúde pública em ascensão, apresentando elevada morbimortalidade e alto índice de complicações que geram consequências de cunho econômico, social e psicológico, além da diminuição da qualidade de vida dos doentes e seus familiares, caracterizandose por um distúrbio metabólico de etiologia múltipla, decorrente de hiperglicemia crônica do comprometimento na produção e/ou utilização de insulina. A doença pode ser classificada em dois grandes subgrupos: DM tipo I, de natureza autoimune ou idiopática ou DM tipo 2, que se caracteriza por defeito na secreção e ação da insulina ${ }^{(1)}$.

Tal doença apresenta diversas complicações crônicas que contribuem para o aumento da morbidade e mortalidade dos pacientes, incluindo aqui a depressão (20\% a 30\%). É sabido que a depressão tem impacto nocivo sobre o controle glicêmico e, por sua vez, o diabetes mal controlado intensifica os sintomas depressivos ${ }^{(2)}$.

Para avaliar os impactos recíprocos entre depressão e diabetes do tipo I e do tipo 2 , foi realizado uma meta-análise sobre a prevalência da depressão em adultos com diabetes e seus impactos recíprocos entre depressão e diabetes do tipo I e do tipo 2. Segundo esses resultados, a depressão está associada à hiperglicemia e a risco aumentado de complicações do diabetes. No sentido oposto, o alívio da depressão associase a melhora significativa do controle glicêmico. A metanálise incluiu 42 estudos e mostrou que a probabilidade de depressão na população diabética foi duas vezes maior do que a da população não diabética. Mostrou ainda que a prevalência de depressão foi significativamente maior em mulheres diabéticas $(28 \%)$ do que em homens $(18 \%)^{(3)}$.

Apesar da importante relação entre depressão e doenças crônicas, não há estudos brasileiros de base populacional que tiveram como objetivo testar a associação entre esses fatores. Uma busca em bases de dados com os descritores "depression", "chronic diseases" e "Brazil" reportou apenas estudos com pacientes hospitalizados ou com doenças específicas, a exemplo de estudos ${ }^{(3)}$, que relatam relação entre quadro depressivo e patologias clinicas como diabetes mellitus.

\section{OBJETIVO}

Relacionar a prevalência de depressão associado ao quadro de DM, tipo 2 de uma população adulta da cidade de pequeno porte no sul do Estado de Minas Gerais.

\section{METODO}

Trata-se de um estudo de análise documental, descritivo, retrospectivo de campo, com amostragem probabilística intencional, realizado em prontuários físicos e eletrônicos do e-sus (sistema de gestão de prontuários eletrônicos do SUS referente à atenção básica) de pacientes portadores de DM 2 , em um município de pequeno porte, localizado no sul do Estado de Minas Gerais, no período de março de 2017 a março de 2018.

Foi utilizado instrumento de coleta de dados com informações sóciodemográficas e clínicas, tais como: sexo, idade, estado civil, escolaridade, atividade laboral, renda média familiar mensal, tempo de DM, complicações da DM, uso de hipoglicemiantes orais e insulina e presença de sintomas depressivos.

Foram considerados como sintomas depressivos a serem extraídos dos prontuários os listados abaixo por período superior a duas semanas e que representaram mudanças no funcionamento prévio do indivíduo:

- Humor deprimido ou perda de interesse ou prazer;

- Perda ou ganho de peso acentuado sem estar em dieta;

- Insônia ou hipersonia quase todos os dias;

- Agitação ou retardo psicomotor quase todos os dias;

- Fadiga e perda de energia quase todos os dias;

- Sentimento de inutilidade ou culpa excessiva ou inadequada quase todos os dias;

- Capacidade diminuída de pensar ou concentrar-se ou indecisão quase todos os dias;

- Pensamentos de morte recorrentes.

Os critérios de inclusão foram: prontuários completos de pacientes com diagnóstico de Diabetes Mellitus tipo 2 e que não usaram qualquer fármaco que pudesse ter como efeito colateral alterações de humor.

O desenvolvimento do estudo ocorreu em conformidade com o preconizado pela Resolução $n^{\circ} 466 / 12$ e 510/16 do Conselho Nacional de Saúde (CNS) e o projeto de pesquisa foi aprovado pelo Comitê Permanente de Ética em Pesquisa com Seres Humanos da Faculdades Mogiana - UNIMOGI (Parecer no. 201.91 I).

Os resultados foram analisados de modo estatístico pela média e desvio padrão das variáveis, bem como a aplicação do teste qui-quadrado e a correlação de Pearson, sendo considerados significantes os resultados com variação de $95 \%(p<0,05)$ para o teste qui-quadrado e acima de 0,5 para a correlação de Pearson.

\section{RESULTADOS}

A cidade em questão conta com população estimada de 3046 indivíduos $^{(4)}$. Desta forma foram avaliados todos os prontuários de sujeitos com Diabéticos tipo 2 (DM 2) do município, perfazendo 317 prontuários ( $10,40 \%$ da população geral com DM 2). Deste total, 91 prontuários foram excluídos, devido critérios de inclusão relacionados ao uso de fármaco; restando 226 prontuários que foram analisados.

Do total de prontuários validos, 68 apresentaram relatos de depressão, com descrição de sintomatologia e medicação prescrita.

Os sujeitos em estudo tinham entre 37 e 88 anos com média de 63 anos $(d p=5,44)$. A maior proporção foi do sexo feminino $(64,66 \%)$, que se encontra na faixa etária de 50 a 70 anos.

Quanto à escolaridade, $40,7 \%$ não completaram o ensino médio e $28,31 \%$ não completaram o ensino fundamental, $39,82 \%$ eram casados e $33,62 \%$, separados ou divorciados (Tabela I)

A presença de sintomas depressivos foi identificada em $30,08 \%$ dos sujeitos com DM 2. Os sintomas depressivos foram mais prevalentes nas mulheres (3I,7\%). A renda mensal de ate dois salários mínimos apresentou relação com depressão em $61,5 \%$ dos sujeitos. A atividade laboral foi significativa em 42,03 dos aposentados e $21,68 \%$ dos trabalhadores rurais. Vale ressaltar que Albertina vive da agricultura e serviço público, com grande parcela da população já aposentada. 
Tabela I - Presença de sintomas depressivos, conforme variáveis sóciodemograficas. Albertina (MG), Brasil, 2018.

\begin{tabular}{|c|c|c|c|c|}
\hline $\begin{array}{c}\text { Variáveis } \\
\text { socioeconômicas } \\
\text { e demográficas }\end{array}$ & $n=226(\%)$ & 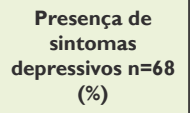 & $\begin{array}{c}* \text { p } \\
\text { significante }\end{array}$ & 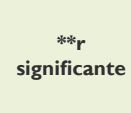 \\
\hline \multicolumn{5}{|l|}{ Sexo } \\
\hline Feminino & $146(64,66)$ & $49(72,05)$ & 0,035 & 0,78 \\
\hline Masculino & $80(35,33)$ & $19(27,95)$ & & \\
\hline \multicolumn{5}{|l|}{ Idade } \\
\hline $301-40$ & $25(11,06)$ & $02(2,94)$ & & \\
\hline $401-50$ & $39(17,25)$ & $04(5,88)$ & & \\
\hline $501-60$ & $55(24,33)$ & $20(29,4 I)$ & 0,042 & 0,54 \\
\hline $601-70$ & $72(31,85)$ & $21(30,88)$ & 0,046 & 0,59 \\
\hline $701-80$ & $21(9,29)$ & $09(13,23)$ & & \\
\hline 80 anos ou mais & $14(6,19)$ & $12(17,64)$ & & \\
\hline \multicolumn{5}{|l|}{ Escolaridade } \\
\hline Analfabeto & $28(12,38)$ & $14(20,58)$ & & \\
\hline $\begin{array}{l}\text { E n s i n o } \\
\text { funda m e n ta l } \\
\text { incompleto }\end{array}$ & $64(28,31)$ & $12(17,64)$ & 0,038 & 0,53 \\
\hline $\begin{array}{l}\text { E n s i n o } \\
\text { fundam e n ta I } \\
\text { completo }\end{array}$ & $16(7,07)$ & $09(13,23)$ & & \\
\hline $\begin{array}{l}\text { Ensino médio } \\
\text { incompleto }\end{array}$ & $92(40,70)$ & $26(38,23)$ & 0,041 & 0,79 \\
\hline $\begin{array}{l}\text { Ensino } \\
\text { completo }\end{array}$ & $12(5,30)$ & $05(7,35)$ & & \\
\hline $\begin{array}{l}\text { S u p e } r i \circ r \\
\text { incompleto }\end{array}$ & $09(3,98)$ & $00(0,00)$ & & \\
\hline Superior completo & $05(2,21)$ & $02(2,94)$ & & \\
\hline \multicolumn{5}{|l|}{ Estado civil } \\
\hline Solteiro & $13(5,76)$ & $09(13,23)$ & & \\
\hline Casado & $90(39,82)$ & II $(16,17)$ & 0,042 & 0,81 \\
\hline Viúvo & $32(14,15)$ & $26(38,23)$ & & \\
\hline $\begin{array}{l}\text { S e p a r a d o / } \\
\text { Divorciado }\end{array}$ & $76(33,62)$ & $17(25,00)$ & 0,031 & 0,78 \\
\hline União estável & $15(6,63)$ & $05(7,35)$ & & \\
\hline \multicolumn{5}{|c|}{ Renda média familiar mensal } \\
\hline Sem renda & $09(3,98)$ & $02(0,88)$ & & \\
\hline $\begin{array}{l}\text { Até } 2 \text { salários } \\
\text { mínimos }\end{array}$ & $139(61,50)$ & $38(16,81)$ & 0,047 & 0,92 \\
\hline $\begin{array}{l}\text { De } 2 \text { a } 5 \text { salários } \\
\text { mínimos }\end{array}$ & $60(26,54)$ & $22(9,73)$ & 0,031 & 0,85 \\
\hline $\begin{array}{l}\text { Acima de } 5 \text { salários } \\
\text { mínimos }\end{array}$ & $18(7,96)$ & $06(2,65)$ & & \\
\hline \multicolumn{5}{|l|}{ Atividade laboral } \\
\hline Motorista & $23(10,17)$ & $08(11,76)$ & & \\
\hline Pedreiro & $18(7,96)$ & $05(7,35)$ & & \\
\hline Costureira & $12(5,30)$ & $02(2,94)$ & & \\
\hline $\begin{array}{l}\text { Funci c oná ri o } \\
\text { público }\end{array}$ & $19(8,40)$ & $02(2,94)$ & & \\
\hline $\begin{array}{l}\text { Trab a l h a d o r } \\
\text { agricultura }\end{array}$ & $49(21,68)$ & $18(26,47)$ & 0,029 & 0,84 \\
\hline Pintor & $07(3,09)$ & OII $(1,47)$ & & \\
\hline Ajudante geral & $03(1,32)$ & 이 $(1,47)$ & & \\
\hline Aposentado & $-42,03$ & $31(45,58)$ & 0,034 & 0,76 \\
\hline
\end{tabular}

*Teste Qui-quadrado $(p<0,05)$ **Correlação de Pearson $(r>0,5)$

Quanto as variáveis clinicas, 37,61\% apresentaram tempo de DM tipo 2 entre 5 a 20 anos e 34, 07\% acima de vinte anos.

Acrescenta-se que em relação a variável complicações clinicas, mais de uma alternativa foi considerada ou mesmo a ausência destas.

A catarata foi a complicação clinica mais predominante, com $20,79 \%$, seguida da dor neuropática (I5,92\%). 79,2\% fazem uso de hipoglicemiantes orais, sem uso concomitante com insulina (84,5 I\%) (Tabela 2).

Tabela 2 - Presença de sintomas depressivos e variáveis clínicas. Albertina (MG), Brasil, 2018.

\begin{tabular}{|c|c|c|c|c|}
\hline Variáveis Clinicas & $\mathrm{n}=226(\%)$ & $\begin{array}{l}\text { Presença de } \\
\text { sintomas } \\
\text { depressivos } \\
\mathrm{n}=68(\%)\end{array}$ & $\begin{array}{c}* p \\
\text { significante }\end{array}$ & $\begin{array}{c}* * *_{r} \\
\text { significante }\end{array}$ \\
\hline \multicolumn{5}{|l|}{ Tempo de DM 2} \\
\hline Até 5 anos & $64(28,31)$ & $04(5,88)$ & & \\
\hline $51-20$ anos & $85(37,61)$ & $29(42,64)$ & 0,048 & 0,58 \\
\hline Acima de 20 anos & $77(34,07)$ & $35(5 \mathrm{I}, 47)$ & 0,022 & 0,89 \\
\hline \multicolumn{5}{|c|}{ Complicações do DM 2} \\
\hline $\begin{array}{l}\text { Ferida membros } \\
\text { inferiores e/ou pé }\end{array}$ & $23(10,17)$ & $04(5,88)$ & & \\
\hline Retinopatia & $14(6,19)$ & $09(13,23)$ & 0,041 & 0,54 \\
\hline Catarata & $47(20,79)$ & $05(7,35)$ & & \\
\hline Glaucoma & II $(4,86)$ & $04(5,88)$ & & \\
\hline Amputações & $08(3,53)$ & $03(4,4 \mathrm{I})$ & & \\
\hline Dor & $36(15,92)$ & $12(17,64)$ & 0,036 & 0,59 \\
\hline Alteração de marcha & $18(7,96)$ & $06(8,82)$ & & \\
\hline Doença renal & $12(5,30)$ & $06(8,82)$ & & \\
\hline Cardiopatia & $24(\mid 0,61)$ & $19(27,94)$ & 0,021 & 0,81 \\
\hline \multicolumn{5}{|c|}{ Uso de hipoglicemiantes orais } \\
\hline Sim & $179(79,20)$ & $51(75,00)$ & & \\
\hline Não & $47(20,80)$ & $17(25,00)$ & & \\
\hline \multicolumn{5}{|l|}{ Uso de Insulina } \\
\hline Sim & $35(15,48)$ & $23(33,82)$ & 0,032 & 0,78 \\
\hline Não & $-84,5 \mid$ & $45(66,18)$ & & \\
\hline
\end{tabular}

*Teste Qui-quadrado $(p<0,05)$ **Correlação de Pearson $(r>0,5)$.

\section{DISCUSSÃO}

O Sistema de Informação da Atenção Básica do Ministério da Saúde (SIAB), no ano de 2013, contava com 33,I milhões de famílias brasileiras cadastradas, abrangendo II4,4 milhões de pessoas ou aproximadamente $57 \%$ da população brasileira (5). Dados complementares da Pesquisa Nacional de Saúde (PNS), realizada em 20I4, evidenciou 7,6\% da população geral brasileira com diabetes mellitus e o estado de Minas Gerais apresentavam média maior que a nacional com II,I\% dos sujeitos com DM.

Esse estudo evidenciou que $10,4 \%$ da população da cidade de Albertina/MG tem quadro de DM 2, ficando esta abaixo da média do estado de Minas Gerais e acima da média nacional, sendo que a maior proporção foi do sexo feminino $(64,66 \%)$, que se encontrava na faixa etária de 50 a 70 anos, concordando com estudos anteriormente realizados ${ }^{(3)}$, na qual a associação de depressão e diabetes foi mais frequente em mulheres (28\%) do que em homens (18\%), embora não houvesse diferença na prevalência de depressão entre o diabetes tipo I e tipo 2 .

A depressão é 50 a $75 \%$ mais frequente em mulheres do que nos homens, e as causas dessas diferenças são desconhecidas, porém, existem possibilidades de relação com influências hormonais, predisposição genética ligada ao cromossomo $X$, ao fato da relação depressiva ser culturalmente mais observável no sexo feminino, por sua maior facilidade em expressar suas emoções e procurar por tratamento, tornando esse transtorno 
mais visível que no sexo masculino ${ }^{(6,7)}$.

Estudos apontam que depressão associada ao diabetes tende a recorrer ao longo dos anos, pois pouco se sabe sobre o curso da depressão associada ao diabetes ${ }^{(8,9)}$, ○ que vem corroborar com os nossos achados pelo fato de apenas 68 indivíduos avaliados apresentarem relatos de depressão, com descrição de sintomatologia e medicação prescrita. Possivelmente há subnotificação diagnóstica pela falta de avaliação diagnóstica para os demais indivíduos.

Em estudo realizado por Felisberto; Saavedra;Santos; Nunes (8), que compreendeu avaliação cinco anos após um ensaio terapêutico para depressão em 25 pacientes com diabetes, encontraram persistência ou recorrência da depressão em 23 (92\%) dos pacientes, com uma média de 4,8 episódios depressivos durante os cinco anos. Durante o primeiro ano após o tratamento, $58,3 \%$ dos pacientes que apresentaram remissão ficaram novamente deprimidos.

Tais dados acima descritos corroboram com estudos em que pacientes diabéticos com depressão apresentaram menor nível educacional e socioeconômico, além de pior suporte social, sendo mais vulneráveis a estressores financeiros e psicossociais $(9,10)$.

Vale ressaltar que a depressão surge mais frequentemente entre pessoas viúvas, divorciadas ou separadas do que entre solteiros e casados, pois a situação de viuvez recente está associada à alta ocorrência de depressão. Além disso, pessoas que moram sozinhas parecem ser mais vulneráveis (II).

Dados obtidos na pesquisa realizada por Werremeyer; Maack; Strand; Barnacle; Petry(12), evidenciam que quanto aos dados relacionados ao estado civil dos participantes, 83,8\% da amostra encontravam-se casados. Assemelham-se tais dados a estudos ${ }^{(13)}$, que demonstram, por meio de seu estudo transversal, um predomínio de participantes casados, pelo percentual também significativo de 55,0\%. Relacionando-se a isto, a literatura ${ }^{(13)}$ que aponta uma associação entre a situação conjugal e os sintomas depressivos e de estresse, no qual estudos revelaram que indivíduos que vivem sem companheiro têm maior predomínio desta sintomatologia.

Em nosso estudo, a presença de sintomas depressivos foi de $30,08 \%$ dos sujeitos com DM 2, e estudos prospectivos (2,14,15), sugerem que a depressão aumente o risco para a ocorrência do diabetes tipo 2. Desta forma, podemos inferir que exista clara associação entre diabetes mellitus e depressão.

Em uma metanálise recente ${ }^{(3)}$, avaliando estudos que utilizaram grupos-controle normais, a prevalência de depressão em pacientes diabéticos era de $11 \%$ a $31 \%$, enquanto que a presença de diabetes aumentava em duas vezes o risco de depressão em relação aos grupos controle.

Além disso, presença de sintomas psiquiátricos a uma doença orgânica que pode ter um efeito devastador sobre a saúde física do indivíduo, podendo influenciar o curso desta doença ${ }^{(16)}$, e que os sintomas depressivos poderiam prejudicar a adesão ao tratamento, piorar o controle metabólico e aumentar o risco de complicações do DM, devido ao fato que a depressão apresenta alterações neuroquímicas e hormonais que teriam efeitos hiperglicemiantes e poderiam acarretar distúrbios no metabolismo glicêmico. Em contrapartida, o DM tem efeitos neuroquímicos sobre os sistemas centrais serotoninérgicos, noradrenérgicos e dopaminérgicos, levando a uma diminuição da função monoaminérgica, à semelhança do que ocorre na depressão(17). A sobreposição de alterações fisiopatológicas de ambas as condições poderia explicar a ocorrência frequente de sintomas depressivos em pacientes diabéticos.

Quanto a relação entre DM2 e complicações crônicas, a catarata foi a mais predominante, com $20,79 \%$, seguida da dor neuropática $(15,92 \%)$, e estudos $(18,19)$, descrevem que a associação entre diabetes tipo 2 e depressão aumenta em $36 \%$ o risco de complicações microvasculares como nefropatia, retinopatia e neuropatia e aumenta em $25 \%$ alterações macrovasculares, como doença vascular periférica, disfunção erétil e principalmente a doença arterial coronariana. Assim, a depressão tem efeito desencadeante e agravante sobre o diabetes tipo 2.

Vale destacar que indivíduos diabéticos com complicações crônicas apresentam níveis de cortisol aumentado, que cronicamente, esses elevados níveis de cortisol geram um estresse neuronal que induz a liberação do fator liberador de corticotrofina pelos neurônios do núcleo paraventricular do hipotálamo. Por sua vez, a corticotrofina via glândula pituitária estimula a liberação de adrenocorticotrofina, que é responsável por estimular a síntese e a liberação de glicocorticóide pelas glândulas adrenais, que podem ter amplos efeitos sobre o humor e as funções comportamentais ${ }^{(20)}$.

\section{CONCLUSÃO}

Conclui-se com esse estudo que ainda que a associação entre diabetes tipo 2 e depressão sejam concretas, não está definida a relação explícita de causa e efeito entre essas duas condições clínicas.

Fica evidente a prevalência de DM 2 acima da média nacional em população geral, com 30,08\% destes apresentando como co-morbidade o quadro depressivo.

Desta forma, sugere-se que políticas públicas devam ser implementadas para diminuição de tais índices no município estudado. Deve-se realizar planejamento e intervenções específicas para a problemática por parte dos gestores municipais. Estudo contribui para revelar situação de prejuízo à saúde que não tinha sido detectado.

\section{REFERENCIAS}

1. Sociedade Brasileira de Diabetes. Diretrizes da Sociedade Brasileira de Diabetes: 2015-2016. São Paulo: Ac farmacêutica, 2016. Disponivel em: https:// www.diabetes.org.br/profissionais/images/docs/ DIRETRIZES-SBD-20I5-2016.pdf

2. American Diabetes Association. Classification and Diagnosis of Diabetes Mellitus. Diabetes Care. 20I5, 38 (suppl): 8- 16. Available from: https://care. diabetesjournals.org/content/38/Supplement_I/S8 Doi: https://doi.org/I0.2337/dc I5-S005

3. Anderson RJ, Freedland KE, Clouse RE, Lustman PJ. The prevalence of comorbid depression in adults with diabetes: a meta-analysis. Diabetes Care. 200I; 24(6): 1069-78. Available from: https://www.ncbi.nlm. nih.gov/pubmed/I I 375373. Doi: https://doi: 10.2337 / diacare.24.6.1069

4. IBGE. Instituto Brasileiro de Geografia Estatística. Estimativas populacionais para os municípios brasileiros em 0 I-07- I8. Rio de Janeiro: IBGE, 20 I8. Disponivel em: <http://www.ibge.gov.br/home/estatistica/populacao/ estimativa20I2/>.

5. Weijun Z, Xu H, Zhao S, Yin S, Wang X, Guo J, et al. Prevalence and influencing factors of co-morbid 
depression in patients with type 2 diabetes mellitus: a General Hospital based study. Diabetol Metab Syndr. 20I5, 7: 60. Available from: https://www.ncbi.nlm.nih. gov/pubmed/26167205 Doi: https://doi.org//0.1/86/ sl3098-015-0053-0

6. Ministério da Saúde. Cadernos de Atenção Básica: Estratégias para o Cuidado da Pessoa com Doença Crônica (Diabetes Mellitus). Brasília- DF, 2013. Disponivel em: http://bvsms.saude.gov.br/bvs/ publicacoes/estrategias_cuidado_pessoa_diabetes_ mellitus_cab36.pdf

7. Gois C, Dias VV, Carmo I, Duarte R, Ferro A, Santos AL, et al. Treatment response in type 2 diabetes patients with major depression. Clin Psychol Psychother. 2014; 2I(I):39-48. Available from: https://www.ncbi.nlm. nih.gov/pubmed/22962030 Doi: https://doi: 10.1002 / cpp. 1817

8. Felisberto VT, Saavedra T, Santos $M$, Nunes $M$. Depression in Type 2 Diabetes Mellitus or Type 2 Diabetes Mellitus in Depression? - A Review. Portuguese J Diabetes. 20 I7; 12 (3): I I 2- I I 7. Available from:Available from: http://www.revportdiabetes.com/ wp-content/uploads/20 I 7/I I/RPD-Vol- I 2-n\%C2\%BA3-Setembro-20 I 7-Artigo-de-Revis\%C3\%A3op\%C3\%Algs-II 2-II7.pdf

9. Lustman PJ, Clouse RE, Freedland KE. Management of major depression in adults with diabetes: Implications of recent clinical trials. Semin Clin Neuropsychiatry. 1998; 3(2):I02-14. Available from: https://europepmc. org/abstract/med/10085197

10. Groot M, Golden S,Wagner J. Psychological conditions in adults with diabetes. Am Psychol. 2016 October; 7I(7): 552-562. Available from: https://www.ncbi.nlm. nih.gov/pubmed/27690484 Doi: https://doi: 10.1037 / a0040408

11. Yokoyama K,YamadaT,Mitani H,Yamada S,Pu S,Yamanashi $\mathrm{T}$, et al. Relationship between hypothalamic- pituitaryadrenal axis dysregulation and insulin resistance in elderly patients with depression. Psychiatry Res. 2015 Apr 30; 226(2-3):494-8. Available from: https://www. ncbi.nlm.nih.gov/pubmed/257579/3 Doi: https://doi: 10.1016/j.psychres.2015.0I.026

12. Werremeyer A, Maack B, Strand MA, Barnacle M, Petry $N$. Disease control among patients with diabetes and severe depressive symptoms. J Prim Care Community Health. 2016; 7(2): I30-I34. Available from: https:// www.ncbi.nlm.nih.gov/pmc/articles/PMC593272 I/ Doi: https://doi: 10.1 I77 / 2150131915627423

13. Boarolli M, Ferreira NC, Bavaresco DV, Ceretta LB, Tuon L, Simões PW, et al. perfil e prevalência de fatores emocionais (estresse e depressão) em pacientes com diabetes tipo 2. Rev Inic Cient, Criciúma. 2015; 13(I). Disponivel em: http://periodicos.unesc.net/ iniciacaocientifica/article/view/2742/2543
14. Braga D, Carli F, Nyland L, Bonamigo E, Bortolini S. Fatores associados à depressão em indivíduos com Diabetes Mellitus. Arq Catarinenses Med. 2017; 46(3): I 18-128. Disponivel em: http://www.acm.org.br/acm/ seer/index.php/arquivos/article/view/3 I 3

15. Bala SS, Jamieson HA, Nishtala PS. Factors associated with inappropriate prescribing among older adults with complex care needs who have undergone the interRAl assessment. Curr Med Res Opin. 2019 May; 35(5):917-923. Available from: https://www. ncbi.nlm.nih.gov/pubmed/30380343 Doi: https://doi: 10.1080/03007995.2018.1543185

16. Silva M dos S, Ramos LR, Lucchesi LM, Lopes GS. Associação entre Alteração da Glicemia de Jejum, Cognição e Capacidade Funcional de Idosos. Revista Enfermagem Atual [Internet]. 9mar. 2019 [citado 27 nov. 2019];86(24). Available from: https:// revistaenfermagematual.com.br/index.php/revista/ article/view/II 0

17. Ferreira D, Daher D, Teixeira E, Rocha I. Repercussão emocional diante do diagnóstico de diabetes mellitus tipo 2 [Emotional impact before the diagnosis of type 2 diabetes mellitus] [Impacto emocional frente ao diagnóstico de diabetes mellitus tipo 2]. Rev Enferm. UERJ. 20I 3; 2 I (I), 4I-46.Available from: https://www.epublicacoes.uerj.br/index.php/enfermagemuerj/article/ view/6346/4519

18. Pols AD, Adriaanse MC, van Tulder MW, Heymans MW, Bosmans JE, van Dijk SE et al. Two-year effectiveness of a stepped-care depression prevention intervention and predictors of incident depression in primary care patients with diabetes type 2 and/or coronary heart disease and subthreshold depression: data from the Step-Dep cluster randomized controlled trial. BMJ Open. 2018; 8(10). Available from: https://www.ncbi. nlm.nih.gov/pmc/articles/PMC62247/8/ Doi: http:// dx.doi.org/ I 0. I | 36/bmjopen-20 I7-0204 I2

19. Linhares BN, Naves VN, Matias RN, Oliveira JCP, Silva DOF. A correlação entre depressão e Diabetes mellitus tipo 2. Rev Med Saude Brasilia. 20 I5; 4(3):34I49. Disponível em: https://portalrevistas.ucb.br/index. php/rmsbr/article/view/6133

20. Prabhakar V, Gupta D, Kanade P, Radhakrishnan. Diabetes-associated depression: The serotonergic system as a novel multifunctional target. Indian J Pharmacol. 2015; 47(I):4-10. Available from: https:// www.ncbi.nlm.nih.gov/pubmed/2582 I 303 Doi: https:// doi: 10.4103 / 0253-7613.150305
Recebido: 2019-09-14

Aceito: $2019-12-10$ 